\title{
Genetically Divergent Highly Pathogenic Avian Influenza A(H5N8) Viruses in Wild Birds, Eastern China
}

\author{
Guimei He, Le Ming, Xiang Li, Yuhe Song, Ling Tang, Min Ma, Jie Cui, Tianhou Wang
}

In late 2020 , we detected 32 highly pathogenic avian influenza $\mathrm{A}(\mathrm{H} 5 \mathrm{~N} 8)$ viruses in migratory ducks in Shanghai, China. Phylogenetic analysis of 5 representative isolates identified 2 sublineages of clade 2.3.4.4b. Each sublineage formed separate clusters with isolates from East Asia and Europe.

$\mathrm{H}$ ighly pathogenic avian influenza (HPAI) $\mathrm{A}(\mathrm{H} 5 \mathrm{Nx})$ clade 2.3.4.4 viruses, which originated from the HPAI A(H5N1) clade 2.3.4 of the A/ Goose/Guangdong/1/96-lineage in China, have spread globally, causing severe disease in poultry and wild birds (1-4). According to the World Health Organization, clade 2.3.4.4 viruses have evolved into 8 subclades, designated as clades 2.3.4.4a-h (https:// www. who.int/influenza/vaccines/virus/202002_zoonotic_vaccinevirusupdate.pdf). In 2013, a novel reassortant $\mathrm{A}(\mathrm{H} 5 \mathrm{~N} 8)$ clade 2.3.4.4b virus was isolated from domestic ducks in eastern China (2); this virus was later detected in Korea and Japan (3). Since 2014, clade 2.3.4.4b viruses have spread to Europe and Africa along the migratory flyways of birds $(4,5)$. These introductions caused large HPAI outbreaks in wild and domestic birds in Europe during the winter of 2016-17 (6). At the same time, wild birds carried clade 2.3.4.4c viruses to North America (4).

In early 2020, outbreaks of clade 2.3.4.4b viruses mainly occurred in Europe (7). Beginning in July 2020, several outbreaks of H5N8 viruses in poultry

Author affiliations: East China Normal University, Shanghai, China (G. He, L. Ming, L. Tang, M. Ma, T. Wang); CAS Key Laboratory of Molecular Virology \& Immunology, Institut Pasteur, Center for Biosafety Mega-Science, Chinese Academy of Sciences, Shanghai (X. Li, Y. Song, J. Cui)

DOI: https://doi.org/10.3201/eid2711.204893 and wild birds were reported in Eurasia, including Kazakhstan, Russia, Poland, England, Netherlands, Korea, and Japan (7-10); outbreaks were not reported in China until October 2020, when clade 2.3.4.4b viruses related to those circulating in Eurasia were detected in 2 dead swans in Mongolia (11). Because eastern China is a major bird migration destination, migratory birds might carry HPAI viruses to this region. We detected $32 \mathrm{H} 5 \mathrm{~N} 8$ viruses of 2 genetically distinct lineages in wild birds in eastern China.

\section{The Study}

On October 31, 2020, we began annual surveillance for avian influenza viruses (AIVs) in migratory birds. As of December 2, 2020, we had collected 612 cloacal and tracheal swab samples from migratory ducks in the Jiuduansha wetland $\left(31^{\circ} 06^{\prime}-31^{\circ} 14^{\prime} \mathrm{N}\right.$, $121^{\circ} 46^{\prime}-122^{\circ} 15^{\prime} \mathrm{E}$ ). This wetland is located at the Yangtze River Estuary and is a major stopover site for migratory birds traveling along the East AsianAustralasian flyway. The birds showed no signs of illness. Reverse transcription PCR detected 32 H5N8 viruses by described procedures (12). We determined the prevalence of $\mathrm{H} 5 \mathrm{~N} 8$ viruses to be $5.2 \%$. These H5N8-positive bird species comprised the common teal (Anas crecca), spot-billed duck (Anas poecilorhyncha), northern pintail (Anas acuta), falcated teal (Anas falcata), and mallard (Anas platyrhynchos). We determined the sequences of the hemagglutinin (HA) and neuraminidase (NA) gene segments of these isolates. We found that 31 isolates had nearly identical HA and NA segments, sharing $99.7 \%-100 \%$ nucleotide sequence identity. We then determined the entire genomic sequences of 5 representative isolates from 5 different host species. We designated these 5 isolates as A/common teal/Shanghai/JDS20103116/2020H5N8 (GenBank accession nos. MW269587-94), A/ northern pintail/Shanghai/JDS20843/2020-H5N8 
(GenBank accession nos. MW362179-86), A/falcated teal/Shanghai/JDS20857/2020-H5N8 (GenBank accession nos. MW362170-7), A/spot-billed duck/ Shanghai/JDS20867/2020-H5N8 (GenBank accession nos. MW362161-8), and A/mallard/ Shanghai/JDS20876/2020-H5N8 (GenBank accession nos. MW357308-15).

Whole-genome sequencing of these $5 \mathrm{H} 5 \mathrm{~N} 8$ viruses revealed that isolate JDS20103116-H5N8 shared a relatively low nucleotide sequence identity $(92.4 \%-$ $97.8 \%$ ) with the other 4 isolates, indicating that these viruses are genetically divergent. BLAST analysis (https:// blast.ncbi.nlm.nih.gov/Blast.cgi) showed that these $5 \mathrm{H} 5 \mathrm{~N} 8$ isolates shared the highest sequence identity $(99.3 \%-100.0 \%)$ with H5N8 viruses isolated in late 2020 from poultry and wild birds in South Korea, Japan, and Europe (including Russia, Netherlands, and England) (Table). To further characterize these 5 isolates, we constructed phylogenetic trees by comparing the sequences of all 8 genomic segments with those in the GISAID database (https://www. gisaid.org) using IQ-TREE (13). We used the general time reversible (GTR) plus F plus G4 model for the HA and polymerase basic 2 protein segments, the transversion e plus G4 model for the matrix protein segment, the K3Pu plus F plus G4 model for the nonstructural protein and NA segments, the transversion plus F plus G4 model for the polymerase acidic protein segment, the GTR plus F plus invariant sites plus
G4 model for the polymerase basic 1 protein segment, and the transition 2 plus $\mathrm{F}$ plus invariant sites plus G4 model for the nucleoprotein segment. We set parameters to $-\mathrm{m}$ (model selection), MFP (model find program), -B (ultrafast bootstrap value), 1,000 bootstraps, $-\mathrm{T}$ (threads for used for tree building), and AUTO (automatically selected number of threads).

Results showed that the isolates belonged to clade 2.3.4.4b and formed 2 distinct genetic sublineages (Figure, https://wwwnc.cdc.gov/ EID/article/27/11/20-4893-F1.htm). The isolate JDS20103116-H5N8 clustered with the isolates found in East Asia (including South Korea and Japan) in late 2020, as well as the isolates found in Europe in late 2019 and early 2020 (14). The other 4 H5N8 isolates clustered with the viruses found in poultry and wild birds in Eurasia (including South Korea, Japan, China, and Europe) in late 2020. The cluster showed high bootstrap support; we proposed the clade to be a novel genotype of the 2.3.4.4b clade (Figure). The topologic structure of trees based on the other gene segments were identical to that of the tree based on the HA gene segment (Appendix Figure, https://wwwnc.cdc.gov/EID/ article/27/11/20-4893-App1.pdf).

The novel genotype of the 2.3.4.4b clade also was closely related to viruses detected in poultry in Iraq in May 2020 and in Egypt in 2019, suggesting that these viruses might be the source of the novel

Table. Nucleotide sequence identity of 5 representative avian influenza A(H5N8) isolates from 5 different host species, Shanghai, China, 2020

\begin{tabular}{|c|c|c|c|c|}
\hline Isolates & Gene segment & Homologous strains $^{*}$ & $\begin{array}{c}\text { GISAID } \\
\text { accession no. }\end{array}$ & Identity, \% \\
\hline \multirow[t]{8}{*}{ JDS20103116 } & Polymerase basic 2 protein & A/duck/Korea/H439/2020 (A/H5N8) & EPI1845982 & 99.9 \\
\hline & Polymerase basic 1 protein & A/duck/Korea/H439/2020 (H5N8) & EPI1845983 & 99.9 \\
\hline & Polymerase acidic protein & A/northern pintail/Hokkaido/M13/2020(H5N8) & EPI1818401 & 99.7 \\
\hline & Hemagglutinin & A/duck/Korea/H439/2020 (A/H5N8) & EPI1845985 & 99.8 \\
\hline & Nucleoprotein & A/duck/Korea/H439/2020 (A/H5N8) & EPI1845978 & 99.3 \\
\hline & Neuraminidase & A/ duck/Korea/H439/2020 (A/H5N8) & EPI1845984 & 99.9 \\
\hline & Matrix protein & $\mathrm{A} /$ chicken/Kagawa/11C/2020(H5N8) & EPI1815028 & 99.8 \\
\hline & Nonstructural protein & A/chicken/Kagawa/11C/2020(H5N8) & EPI1815027 & 99.4 \\
\hline \multirow{16}{*}{$\begin{array}{l}\text { JDS20843, } \\
\text { JDS20843, } \\
\text { JDS20867, } \\
\text { JDS20876 }\end{array}$} & Polymerase basic 2 protein & A/wild duck/Korea/H331/2020 (H5N8) & EPI1846695 & 99.6 \\
\hline & & A/spot-billed duck/Korea/WA1000/2020 (H5N8) & EPI1846695 & 99.6 \\
\hline & Polymerase basic 1 protein & A/quail/Korea/H440/2020 (H5N8) & EPI1846512 & 99.7 \\
\hline & & A/chicken/Korea/H440/2020 (H5N8) & EPI1845991 & 99.7 \\
\hline & Polymerase acidic protein & A/chicken/Omsk/0119/2020 (H5N8) & EPI1813381 & 99.9 \\
\hline & & A/domestic duck/kazakhstan/1-274-20-B/2020 (H5N8) & EPI1811610 & 99.9 \\
\hline & Hemagglutinin & A/chicken/Korea/H544/2020 (H5N8) & EPI1850622 & 100.0 \\
\hline & & A/chicken/Korea/H001/2021 (H5N8) & EPI1846522 & 100.0 \\
\hline & Nucleoprotein & A/mallard/Kagoshima/ KU-d89/2021 (H5N8) & EPI1846675 & 99.9 \\
\hline & & A/spot-billed duck/Korea/Wa1000/2020 (H5N8) & EPI1846697 & 99.9 \\
\hline & Neuraminidase & A/wild duck/Korea/H331/2020 (H5N8) & EPI1850682 & 99.9 \\
\hline & & A/chicken/Korea/H002/2021 (H5N8) & EPI1846529 & 99.9 \\
\hline & Matrix protein & A/wild bird/Korea/H496-3/2020 (H5N8) & EPI1857465 & 100.0 \\
\hline & & A/chicken/Korea/H544/2020 (H5N8) & EPI1850662 & 100.0 \\
\hline & Nonstructural protein & A/Greylag_goose/England/033100/2020 (A/H5N8) & EPI1837929 & 99.8 \\
\hline & & A/turkey/Omsk/0003/2020 (H5N8) & EPI1846695 & 99.8 \\
\hline
\end{tabular}

*List comprises the most or the 2 most homologous viruses available in GISAID (https://www.gisaid.org) for each gene segment. 
genotype. After the outbreaks in Iraq, clade 2.3.4.4b viruses were detected in backyard poultry in Russia in late July 2020 and in wild birds in Russia and Kazakhstan in September 2020. In October 2020, those viruses were also prevalent among birds traveling along various migratory flyways of Europe and Asia (7-10). We speculate that these viruses circulated among domestic birds in Egypt and then among migratory birds in Russia before emerging in Eurasia in late 2020. Because of the lack of surveillance data at breeding sites in 2019 and early 2020, the transmission routes of these viruses remain unclear. In February 2021, an avian influenza H5N8 infection was reported in a person in Russia. The causative virus, designated A/Astrakhan/3212/2020H5N8, belonged to the 2.3.4.4b clade. These observations suggest that the $\mathrm{H} 5 \mathrm{~N} 8$ viruses in this novel genotype of 2.3.4.4b clade could infect a wide range of hosts and might spread globally, as did previous H5N8 outbreaks that spread from Asia to Europe and North America in 2014 (15).

Molecular analysis of HA cleavage sites demonstrated that the $5 \mathrm{H} 5 \mathrm{~N} 8$ isolates contain multiple basic amino acids, PLREKRRKR/GL, which are characteristic of HPAI viruses. The HA1 receptor-binding sites of all $5 \mathrm{H} 5 \mathrm{~N} 8$ isolates have amino acid residues Q226 and G228 (H3 numbering), indicative of an avian-like (a2, 3-SA) receptor-binding preference. We documented 2 new amino acid substitutions, T140A and N236D (H3 numbering), in the HA protein of the novel genotype of the 2.3.4.4b clade. The significance of these 2 new mutations remains undetermined. We did not find the E627K and D701N residues in the polymerase basic 2 protein, suggesting that the viruses have not adapted to mammal hosts.

\section{Conclusions}

During our annual surveillance, we detected 32 H5N8 HPAI viruses from migratory ducks without signs of illness in Shanghai, China. Results of phylogenetic analyses of 5 representative isolates showed that they belonged to 2 sublineages of $\mathrm{H} 5 \mathrm{~N} 8$ viruses circulating in this region. Some isolates clustered with a novel genotype of 2.3.4.4b clade that was identified in Europe and East Asia in late 2020. The detection of these H5N8 AIVs in asymptomatic migratory birds support the hypothesis that free-living wild birds play a crucial role in the dissemination of these viruses. More active surveillance is needed to detect new AIVs, especially in the breeding grounds and migratory sites of various birds. Because of their high genetic diversity, new AIVs might pose a substantial threat to global health.

\section{Acknowledgments}

We thank Shanghai Forestry Station for assistance in field sampling and all investigators who submitted avian influenza sequences to the GISAID database (https://www.gisaid.org), making this study possible. We greatly appreciate Ron A.M. Fouchier, Bianca Zecchin, Elliot Whittard, Ivan Sobolev, Ivan M. Susloparov, Vasily Evseenko, Katalin Szentpáli-Gavallér, Edyta Swieton, and Jacqueline King for permission to use the sequences they submitted to GISAID.

This work was supported by grants from the National Key Research and Development Program (grant no. 2016YFD0500201), Shanghai Science and Technology Committee (grant no. 18DZ2293800), and Shanghai Wildlife-Borne Infectious Disease Monitoring Program.

\section{About the Author}

Dr. He is an associate professor at East China Normal University, Shanghai, China. Her primary research interests are molecular epidemiology of avian influenza viruses in wild birds and pathogenesis of acute lung injury induced by influenza viruses.

\section{References}

1. Gu M, Liu W, Cao Y, Peng D, Wang X, Wan H, et al. Novel reassortant highly pathogenic avian influenza (H5N5) viruses in domestic ducks, China. Emerg Infect Dis. 2011;17:1060-3. https://doi.org/10.3201/eid/1706.101406

2. Wu H, Peng $X, X u$ L, Jin C, Cheng L, Lu X, et al. Novel reassortant influenza $\mathrm{A}(\mathrm{H} 5 \mathrm{~N} 8)$ viruses in domestic ducks, eastern China. Emerg Infect Dis. 2014;20:1315-8. https:// doi.org/10.3201/eid2008.140339

3. Lee YJ, Kang HM, Lee EK, Song BM, Jeong J, Kwon YK, et al. Novel reassortant influenza A(H5N8) viruses, South Korea, 2014. Emerg Infect Dis. 2014;20:1087-9. https://doi.org/10.3201/eid2006.140233

4. Lee DH, Torchetti MK, Winker K, Ip HS, Song CS, Swayne DE. Intercontinental spread of Asian-origin H5N8 to North America through Beringia by migratory birds. J Virol. 2015;89:6521-4. https://doi.org/10.1128/JVI.00728-15

5. Bouwstra R, Heutink R, Bossers A, Harders F, Koch G, Elbers A. Full-genome sequence of influenza A(H5N8) virus in poultry linked to sequences of strains from Asia, the Netherlands, 2014. Emerg Infect Dis. 2015;21:872-4. https://doi.org/10.3201/eid2105.141839

6. Napp S, Majó N, Sánchez-Gónzalez R, Vergara-Alert J. Emergence and spread of highly pathogenic avian influenza A(H5N8) in Europe in 2016-2017. Transbound Emerg Dis. 2018;65:1217-26. https://doi.org/10.1111/tbed.12861

7. Lewis NS, Banyard AC, Whittard E, Karibayev T, Al Kafagi T, Chvala I, et al. Emergence and spread of novel H5N8, H5N5 and H5N1 clade 2.3.4.4 highly pathogenic avian influenza in 2020. Emerg Microbes Infect. 2021;10:14851. https://doi.org/10.1080/22221751.2021.1872355

8. Isoda N, Twabela AT, Bazarragchaa E, Ogasawara K, Hayashi H, Wang ZJ, et al. Re-invasion of H5N8 high pathogenicity avian influenza virus clade $2.3 .4 .4 \mathrm{~b}$ in Hokkaido, Japan, 2020. Viruses. 2020;12:1439. 
https:/ / doi.org/10.3390/v12121439

9. Jeong S, Lee DH, Kwon JH, Kim YJ, Lee SH, Cho AY, et al. Highly pathogenic avian influenza clade 2.3.4.4b subtype H5N8 virus isolated from mandarin duck in South Korea, 2020. Viruses. 2020;12:1389. https:// doi.org/10.3390/ v12121389

10. Baek YG, Lee YN, Lee DH, Shin JI, Lee JH, Chung DH, et al. Multiple reassortants of H5N8 clade 2.3.4.4b highly pathogenic avian influenza viruses detected in South Korea during the winter of 2020-2021. Viruses. 2021;13:490. https:/ / doi.org/10.3390/v13030490

11. Li X, Lv X, Li Y, Peng P, Zhou R, Qin S, et al. Highly pathogenic avian influenza A (H5N8) virus in swans, China, 2020. Emerg Infect Dis. 2021;27:1732-4. https:/ / doi. org/10.3201/eid2706.204727

12. Tang L, Tang W, Ming L, Gu J, Qian K, Li X, et al. Characterization of avian influenza virus H10-H12 subtypes isolated from wild birds in Shanghai, China from 2016 to 2019. Viruses. 2020;12:1085. https:/ / doi.org/10.3390/ v12101085

13. Minh BQ, Schmidt HA, Chernomor O, Schrempf D, Woodhams MD, von Haeseler A, et al. IQ-TREE 2: new models and efficient methods for phylogenetic inference in the genomic era. Mol Biol Evol. 2020;37:1530-4.

https://doi.org/10.1093/molbev/msaa015

14. Świętoń E, Fusaro A, Shittu I, Niemczuk K, Zecchin B, Joannis T, et al. Sub-Saharan Africa and Eurasia ancestry of reassortant highly pathogenic avian influenza A(H5N8) virus, Europe, December 2019. Emerg Infect Dis. 2020;26:1557-61. https:// doi.org/10.3201/eid2607.200165

15. Global Consortium for H5N8 and Related Influenza Viruses. Role for migratory wild birds in the global spread of avian influenza H5N8. Science. 2016;354:213-7. https://doi.org/10.1126/science.aaf8852

Address for correspondence: Tianhou Wang, School of Life Sciences, East China Normal University, no. 3663, North Zhongshan Rd, Shanghai, China; email: thwang@bio.ecnu. edu.cn; Jie Cui, CAS Key Laboratory of Molecular Virology \& Immunology, Institute Pasteur, Center for Biosafety MegaScience, Chinese Academy of Sciences, Shanghai, China; email: jcui@ips.ac.cn

\section{EID Podcast: Unusual Outbreak of Rift Valley Fever in Sudan}

Rift Valley Fever is a devastating disease that can cause bleeding from the eyes and gums, blindness, and death. In 2019, an outbreak of this vectorborne disease erupted among people and animals in a politically volatile region of Sudan. This outbreak broke traditional patterns of Rift Valley Fever, sending scientists scrambling to figure out what was going on and how they could stop it.

In this EID podcast, Dr. Ayman Ahmed, a scientist at the University of Texas Medical Branch and a lecturer at the Institute of Endemic Diseases in Sudan, discusses the intersection of political unrest and public health.

Visit our website to listen: http://go.usa.gov/xAC5H 